Ann. Sci. forest., 1982, 39 (2), 179-185

\title{
Conservation pendant 5 ans de graines de peupliers noirs (Populus nigra L.)
}

\author{
Claudine MULLER * et Eric TEISSIER du CROS ** \\ avec la collaboration technique d'Elyane LAROPPE * \\ et de Hervé DUVAL ** \\ * I.N.R.A., Laboratoire de graines, Centre de Recherches forestières de Nancy, \\ Champenoux, $F 54280$ Seichamps \\ ** I.N.R.A., Station d'Amélioration des Arbres forestiers, \\ Centre de Recherches d'Orléans \\ Ardon, F 45160 Olivet
}

\begin{abstract}
Résumé
Sur le strict plan de la germination, les graines de Populus nigra peuvent se conserver pendant au moins 5 ans si leur teneur en eau est de 7 à 8 p. 100 et si elles sont stockées en petites ampoules scellées sous vide partiel à une température de $+4{ }^{\circ} \mathrm{C}$. Cependant le taux de survie de semis repiqués en pots individuels après 1 année de conservation des graines est en faveur de $-15^{\circ} \mathrm{C}(72 \mathrm{p}, 100)$, mais il est encore suffisant à $-5^{\circ} \mathrm{C}$ (55 p. 100).
\end{abstract}

\section{I. - Introduction}

La connaissance précise des conditions de conservation des graines, répond, pour la majorité des peupliers, à des besoins de la recherche et trouve son intérêt dans les programmes d'amélioration :

- rassemblement de «matériel sauvage» dans les peuplements naturels pour l'appréciaton de la variabilité, la constitution de banques de gènes, les échanges entre instituts de recherche,

- réception de graines de l'étranger à des époques où il est impossible de les semer : échanges entre hémisphère nord et hémisphère sud, notamment,

- croisements contrôlés répondant à un plan de croisement pouvant s'échelonner sur plusieurs années.

La graine de peuplier est petite et légère : 0,012 à $0,130 \mathrm{mg}$ selon les espèces ; elle perd relativement rapidement sa faculté germinative à l'air libre : 2 à 4 semaines. Un séchage de 2 à 8 jours à température ambiante (teneur en eau voisine de 6 p. 100), 
suivi d'une conservation en tubes scellés au voisinage de $0^{\circ} \mathrm{C}$, permet le maintien de la viabilité plusieurs mois, voire plusieurs années (U.S.D.A., 1974). JoHNSON (1946) donne une fourchette de teneur en eau plus large pour Populus tremuloïdes et $P$. grandidentata : 7 à 20 p. 100 . Asakawa (1980) montre que des graines de $P$. maximowiczii se conservent plus longtemps lorsqu'elles ont un faible taux d'humidité relative. La mise sous vide utilisée par MARCET (1957) et RoHMEder (1941) ne modifie en rien la faculté germinative. Par ailleurs, Benson \& HARder (1972) ont montré que divers lots de graines de trembles américains germaient de 13 à 93 p. 100 après quatre années en dessicateur à $-24{ }^{\circ} \mathrm{C}$ contre seulement 0 à 51 p. 100 à $+4{ }^{\circ} \mathrm{C}$. Enfin SimaK (1980) recommande pour Salix caprea et Populus tremula la conservation des graines sans leur coton.

Pour Populus nigra quelques précisions sont données par ZsuFFA en 1974 : les graines de cette espèce peuvent être conservées plusieurs années à basse température $\left(-3\right.$ à $\left.-8^{\circ} \mathrm{C}\right)$ et faible humidité $(6 \mathrm{p} .100)$. Ces précisions nous sont malheureusement parvenues après la mise en route de notre propre étude.

Cette étude avait pour objectif de préciser le rôle de la maturité, de la teneur en eau, de la température et de la durée de conservation sur la faculté germinative de graines de Populus nigra.

\section{II. - Matériel et méthode}

L'essai a porté sur 2 lots de graines de Populus nigra récoltées le 29 mai 1974 sur 2 arbres de peuplements naturels des Alpes françaises, présentant des stades de maturation différents à la récolte :

\section{Arbre 1}

Capsules vertes, fermées ou rarement déhiscentes, graines vert blanchâtre.

\section{Arbre 2}

Capsules brunes, largement ouvertes, coton apparent, aggloméré sur les grappes, graines blanc neige.

Nous avons étudié l'influence des facteurs suivants sur la conservation :

- mode de séchage : à l'air ou en dessicateur sur actigel ; durée 4 jours,

- mode de mise sous vide : trompe à eau (pression obtenue de 10 à $25 \mathrm{~mm}$ de mercure selon le débit), pompe à membrane (18 $\mathrm{mm}$ de mercure). Ces deux méthodes n'ayant pas donné de résultats différents, les valeurs des modalités correspondantes ont été regroupées,

- température de conservation : $+4{ }^{\circ} \mathrm{C},-5^{\circ} \mathrm{C},-15^{\circ} \mathrm{C}$.

Les tests de germination suivants ont été réalisés : avant séchage, après séchage, après mise sous vide, puis, après $12,24,36$ et 60 mois de conservation.

Pendant leur transport et jusqu'à leur conditionnement les graines ont été stockées en sacs de papier kraft avec capsules et coton. La conservation des graines débarrassées 
des capsules et du coton a été réalisée en ampoules scellées de $2 \mathrm{~cm}^{3}$ pour chacune des modalités prévues.

Les tests de germination ont porté sur 2 répétitions de 50 graines de chacune des ampoules. Ils ont été effectués à $20^{\circ} \mathrm{C}$, en boîtes de Pétri, sur papier filtre humidifié à l'eau distillée. La durée du test a été fixée à 10 jours selon les normes de l'Association Internationale d'Essais de Semences (I.S.T.A.).

Ont été considérées comme viables des graines ayant donné naissance à 2 cotylédons et une radicule. Les plantules couchées sont incluses dans ce décompte. En effet, le substrat en papier filtre ne nous a pas paru favorable à la stabilité des tous jeunes semis.

Après douze mois de conservation, 10 semis viables par boîte de Pétri ont été repiqués en pots individuels. Leur survie et leur hauteur totale ont été observées en octobre de la même année.

\section{III. - Résultats}

\subsection{Mode de séchage et teneur en eau}

Curieusement la teneur en eau des graines avant séchage était moins élevée pour l'arbre 1 ( 9 p. 100) que pour l'arbre $2(24$ p. 100) dont l'état de maturité était pourtant le plus avancé. En 4 jours, ces teneurs passent à 10 p. 100 pour les 2 lots par séchage à l'air ambiant et à respectivement 7 et 8 p. 100 pour les lots 1 et 2 sous dessicateur. Pour la suite de l'essai l'effet du mode de séchage a été confondu avec la teneur en eau. Seule cette dernière caractéristique sera retenue dans la discussion.

\subsection{Interaction teneur en eau-température}

Les figures 1 et 2 font apparaître que toutes températures confondues la conservation est meilleure aux teneurs en eau de 7 à 8 p. 100 qu'à 10 p. 100 . Un autre résultat classique apparaît aussi, c'est l'interaction teneur en eau-température. Si la teneur en eau est faible (figure 1), la température de conservation ne semble pas avoir d'influence (figure 2), l'effet de la température se manifeste par une diminution de la faculté germinative à $+4^{\circ}$, particulièrement nette et, dès le $24^{\circ}$ mois, pour le lot 2 .

\subsection{Stade de maturité des graines}

Ce facteur est confondu ici avec l'«effet arbre» dont il ne faut pas négliger l'importance. Quoi qu'il en soit, dès le départ de l'essai, les graines de l'arbre 1 (capsules fermées) ont germé mieux que celles de l'arbre 2 (capsules largement ouvertes). A faible taux d'humidité (figure 1) la différence de viabilité des graines entre les deux lots ne varie pratiquement pas du $24^{\mathrm{e}}$ au $60^{\mathrm{e}}$ mois de conservation. Le compor- 
tement moins bon du lot 2 n'est vraisemblablement pas dû à une immaturité puisque précisément ce lot était à un stade plus avancé que le lot 1 .

Il est donc probable qu'il faille considérer la forte teneur en eau initiale ( 24 p. 100) du lot 2 comme responsable de cette différence, soit par un échauffement pendant le transport et jusqu'au conditionnement, soit par une mise en route du processus de germination (la graine de Populus nigra n'est pas dormante).

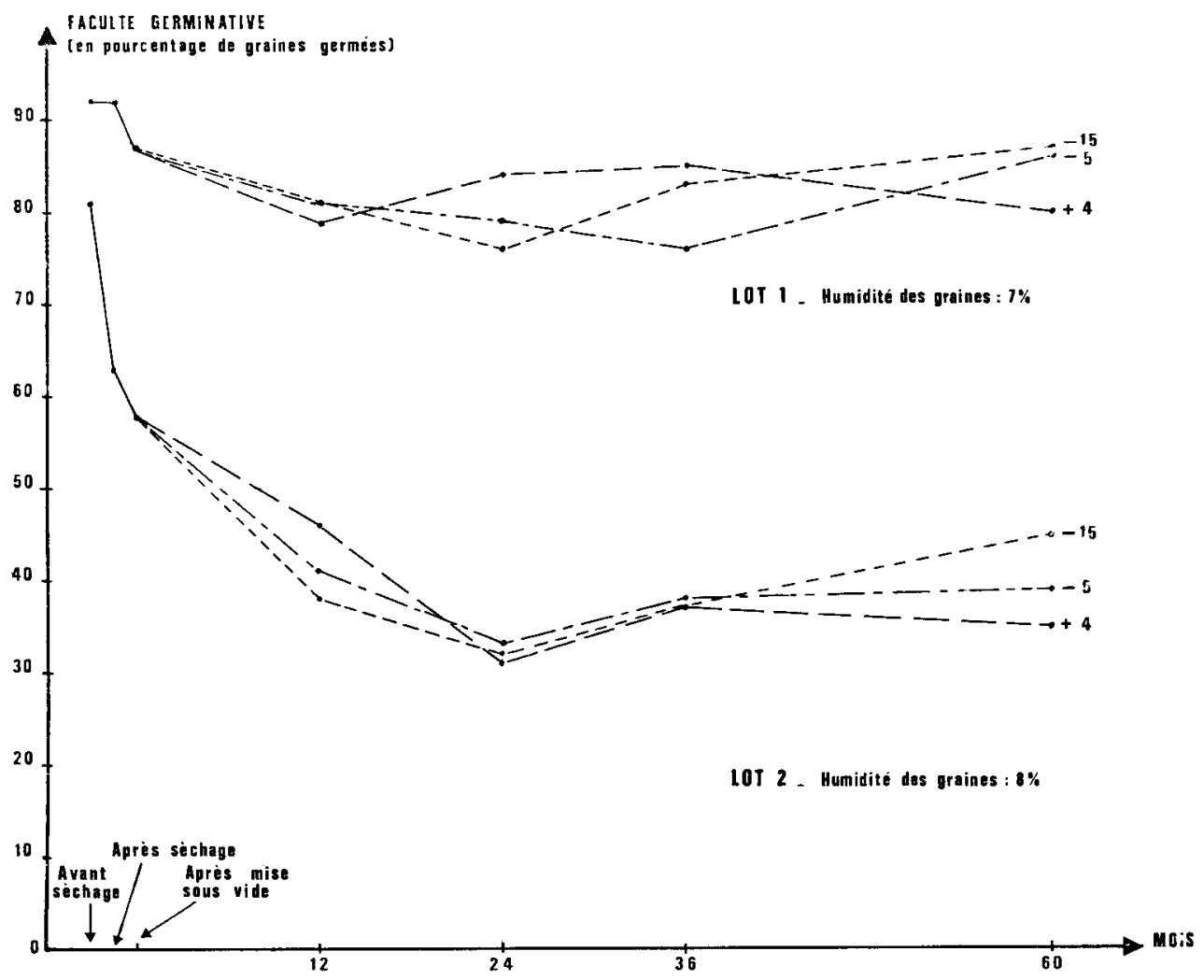

FiguRE 1

Evolution de la faculté germinative sur 60 mois pour deux lots de graines de Populus nigra. Humidité des graines 7 à 8 p. 100

Evolution of germinative capacity over 60 months for two seed lots of Populus nigra. Moisture content 7 to 8 p. 100

\subsection{Survie et croissance des plants}

Le tableau 1 donne les valeurs de ces deux caractères pour les différentes modalités de l'essai (observations réalisées 5 mois après repiquages de 10 semis par 
boîte de Pétri après 12 mois de conservation des graines). On ne constate pas de différence significative liée à la teneur en eau après séchage. Par contre la température de conservation joue un rôle net sur la reprise mais peu sensible sur la hauteur en faveur des températures les plus basses. Les deux lots ont une survie différente, en faveur du lot 2 à maturité plus avancée.

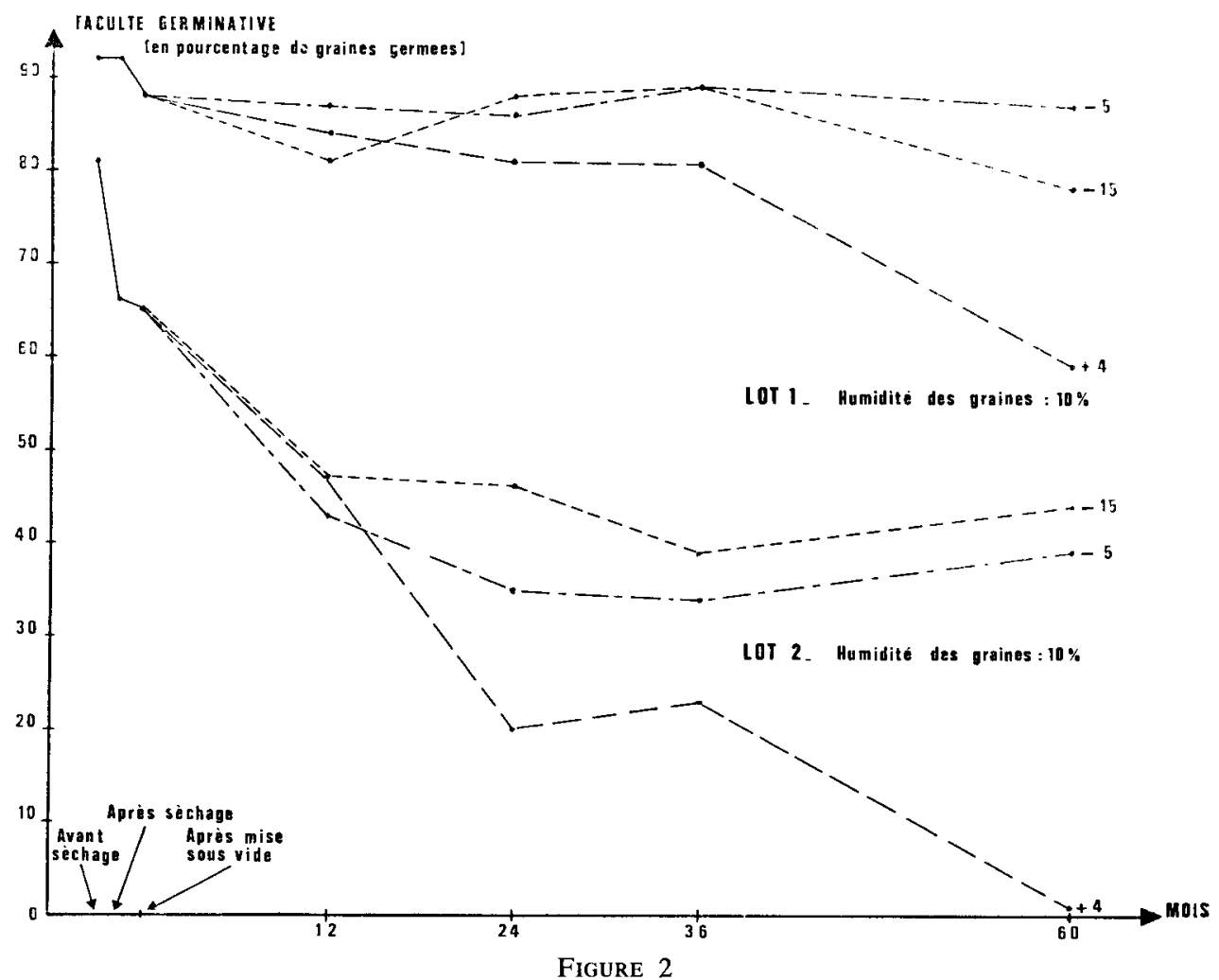

Evolution de la faculté germinative sur 60 mois pour deux lots de graines de Populus nigra. Humidité des graines $10 \mathrm{p} .100$

Evolution of germinative capacity over 60 months for two seed lots of Populus nigra. Moisture content 10 p. 100

\section{IV. - Discussion}

Cette étude confirme qu'il est possible de conserver des graines de Populus nigra avec maintien d'une bonne faculté germinative, pendant plusieurs mois voire plusieurs années. L'essai n'avait été prévu que pour 5 ans, mais l'allure des courbes (figure 1 et température négative de la figure 2) laisse supposer que cette conservation pourrait se poursuivre plus longtemps. 


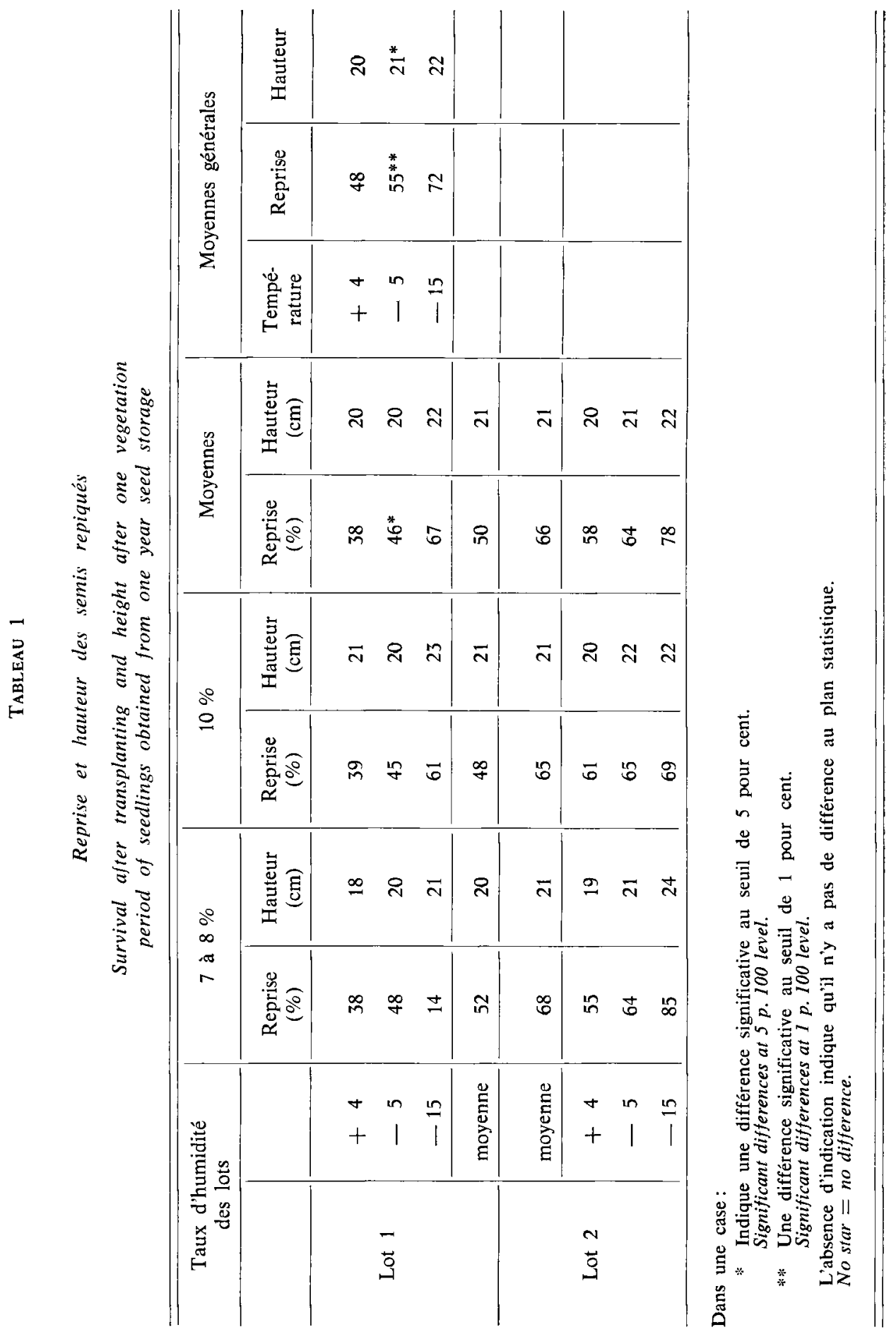


L'essai comportait quelques lacunes, notamment la conservation de graines sèches en capsules scellées, mais à pression atmosphérique, ou encore un séchage accéléré par chauffage. Le nombre de lots était trop faible ce qui n’a pas permis de dissocier l'effet maturité des graines de l'effet arbre.

Néanmoins, il semble d'ores et déjà possible de proposer une technique de conservation satisfaisante :

- séchage jusqu'à une teneur en eau de 7 à 8 p. 100 ,

- mise sous vide relatif en ampoules scellées,

- température de conservation : sous réserve d'une déshydratation conforme à la prescription ci-dessus, la température de +4 "C semble tout à fait satisfaisante. C'est donc sur l'obtention de cette teneur en eau qu'il convient d'insister. Resterait à confirmer l'influence, semble-t-il favorable à la reprise, des conservations à températures plus basses $\left(-5^{\circ} \mathrm{C}\right.$ à $\left.-15^{\circ} \mathrm{C}\right)$, ce qui pourrait inciter à les adopter.

Reçu pour publication le 7 juillet 1981.

\section{Summary}

Five year storage of Populus nigra seeds

Populus nigra seeds may be stored for at least 5 years if their moisture content is around 7 to 8 p. 100 and if they are put in small sealed vials under partial vacuum at $+4^{\circ} \mathrm{C}$. Nevertheless survival of seedlings transplanted in single peat pots after one year storage is higher at a temperature of $-15^{\circ} \mathrm{C}(72 \mathrm{p} .100)$, but quite sufficient at -5 " $\mathrm{C}$ (55 p. 100).

\section{Références bibliographiques}

Asakawa S., 1980. Storage of Populus maximowiczii seeds IUFRO Working Party S 20106 , Seed Problems. International symposium on forest tree seed storage. Petawawa, Canada, 7 p.

Benson M.K., Harder M.L., 1972. Storage of aspen seed. Genetics and physiology notes. No. 11. The Institute of Paper Chemistry, Appleton, Wisconsin, U.S.A. 4 p.

JoHnson L.P.V., 1946. Effect of humidity on the longevity of Populus and Ulmus seeds in storage. Can. J. Res., 124, 298-302.

Marcet E., 1957. Zur Aufbewahrung von Aspensamen. J. for. Suisse, 108 (5), 270-273.

Rohmehder E., 1941. Die Vermehrung der Pappeln durch Samen. Forstarchiv 17 (5/6), 73-80.

Simak M., 1980. Germination and storage of Salix caprea L. and Populus tremula L. seeds. IUFRO Working Party S 20106 , Seed Problems. International symposium on forest tree seed storage. Petawawa, Canada, $18 \mathrm{p}$.

USDA Forest Service, 1974. Seeds of the woody plants in the United States. U.S. Dept of Agric. Handbook, no. 450, 883 p.

Zsuffa L., 1974. The genetics of Populus nigra L. Ann. for., 6/2, 53 p. 